\title{
Effects of Nitrogen on Seedling Growth of Wheat Varieties under Salt Stress
}

\author{
Muhi Eldeen Hussien Ibrahim ${ }^{1,2}$, Xinkai Zhu ${ }^{1}$, G. Zhou ${ }^{1} \&$ Eltayib H. M. A. Abidallhaa ${ }^{1,3}$ \\ ${ }^{1}$ Jiangsu Key Laboratory of Crop Genetics and Physiology, Co-Innovation Center for Modern Production \\ Technology of Grain Crops, Yangzhou Wheat Research Institute, Yangzhou University, Yangzhou, China \\ 2 Department of Crop science, College of Agricultural Studies, Sudan University of Science \& Technology, \\ Khartoum, Sudan \\ ${ }^{3}$ Department of Forest Management, Faculty of Forestry, University of Khartoum, Khartoum, Sudan \\ Correspondence: Xinkai Zhu, Jiangsu Key Laboratory of Crop Genetics and Physiology, Co-Innovation Center \\ for Modern Production Technology of Grain Crops, Yangzhou Wheat Research Institute, Yangzhou University, \\ Yangzhou 225009, China. E-mail: xkzhu@yzu.edu.cn
}

\begin{tabular}{|c|c|c|}
\hline 016 & ed: August 16, 2016 & Online Published: September 15, 2016 \\
\hline 39/jas y & URL $\cdot \mathrm{httn} \cdot / / \mathrm{dx}$ doi orc & 131 \\
\hline
\end{tabular}

\begin{abstract}
Wheat growth is hampered by various environmental stresses including salinity. The aim of the present study was to evaluate the nitrogen effect on seedling emergence and growth under salinity conditions. For this reason the seeds of wheat (Triticum aestivum L.) varieties Argine, and Elnilein from Sudan and Xumai 30, and Yang 10-13 from China were cultured under four $\mathrm{NaCl}$ solutions containing $(0,100,150$, and $200 \mathrm{mM} \mathrm{NaCl})$ and three nitrogen $(\mathrm{N})$ fertilizer levels $(\mathrm{N} 0=0, \mathrm{~N} 1=105$, and $\mathrm{N} 212=210 \mathrm{~kg} \mathrm{~N} / \mathrm{h})$. Emergence percentage and early growth were determined. There were significant differences among salinity and $\mathrm{N}$ levels for emergence percentage shoot and root length, dry weight, salt tolerance index, and seedling vigor index. At all salinity levels, the varieties showed similar salt resistance, but each responded differently. Nitrogen affected positively on the seedling characteristics under saline soil. Elnilein had a better emergence percentage, shoot and root length, dry weight, salt tolerance index, and seedling vigor index than the other varieties. Elnilein is recommended for saline soils. We suggest that a simple seedling test would be a useful selection tool in order to develop productive new wheat lines on saline soils.
\end{abstract}

Keywords: nitrogen, salinity, seedling growth, wheat

\section{Introduction}

Salinity is one of the main abiotic stresses and playing main role in decreasing on yield and of many crops and degradation of soil in the world (Meloni et al., 2008). The reduction in growth observed in many plants subjected to salinity stress is often correlated with salt-induced osmotic effect, nutrient deficiency, specific ion toxicity, or their combinations (Munns, 2002). Given the large areas of salt-affected soils in the world, it is not surprising that plants have evolved mechanisms to regulate the accumulation of $\mathrm{NaCl}$ (Munns \& Tester, 2008). Nitrogen is the most limiting nutrient in crop production, and its efficient use to increase food production is more than any other input; however, much use of $\mathrm{N}$ may cause environmental concerns such as nitrate leaching, eutrophication, and greenhouse gas's emissions and reduce crop yield (Malhi et al., 2001). Therefore, proper use of $\mathrm{N}$ is critical to optimize crop yield and minimize environmental damage. It has been estimated that $40 \%-60 \%$ of $\mathrm{N}$-applied is taken up by wheat, which decreases as the $\mathrm{N}$-input increases, resulting in higher residual soil $\mathrm{N}$ that can be readily leached (Guarda et al., 2004).

Seed germination and early seedling growth determine stand establishment and yield potential. Seedlings are the most vulnerable stage in the life cycle of plants, and germination determines when and where seedling growth begins (Llanes et al., 2005), and soil salinity affected plants at seedling stage much higher than other plant growth stages because seed germination usually occurs in the uppermost soil layers which accumulate soluble salts as a result of evaporation and capillary rise of water (Almansouri et al., 2001). In addition, the mechanisms for salt tolerance that have been identified during vegetative growth, for example, salt uptake combined with vacuolar compartmentalization (Qiu et al., 2007) or $\mathrm{Na}+$ bound in starch granules (Kanai et al., 2007) are 
energies intensive and reduce carbohydrate reserves available to the seedling. Thus, although excess sodium and chloride ion imbalance has a deleterious effect on many cellular systems at all growth stages (Zhu et al., 1997) metabolic tolerance to these ions is more important during germination than at later life stages, due to the limited carbohydrate reserves available in the seed (Zhang et al., 2014).

Wheat (Triticum aestivum L.) is a major food crop for more than one-third of the world population and is the staple food in Asia (Shirazi et al., 2001). Wheat cultivation in Sudan has been expanding in the last decades to latitudes lower than $15^{\circ} \mathrm{N}$ as a winter crop, occupying the largest area in Sudanese irrigated schemes, and it is now the second most important cereal crop after sorghum in the country (Saunders \& Hettel, 1994). China is the world's greatest wheat producer and consumer, and for many years it is the biggest importer too. Seed germination and seedling growth of wheat, like many other major crops, are negatively affected by drought (Passioura \& Gardner, 1990) and salinity stress (Hampson \& Simpson, 1990) Salinity compounds the detrimental effects of drought on germination and seedling growth, by inhibiting water uptake, through toxic effects of $\mathrm{Na}$ and $\mathrm{Cl}$ ions, or by a combination of the two (Zhang et al., 2010). Wheat is generally not considered a halophyte, but because of its importance as a food crop worldwide, selection of varieties that have some level of salt resistance, particularly during germination, is highly desirable.

In this study, we hypothesize that deleterious effects of salinity could be ameliorated by nitrogen fertilizer, and will help to promote the early seedling growth of wheat for better crop establishment. The objective of this study are to examine the possibility of alleviating salt stresses by using nitrogen fertilizer and to examine the difference in salt resistance under difference nitrogen levels during seedling stage, among wheat varieties bred in China and Sudan and currently grown on salt-stressed soils.

\section{Materials and Methods}

\subsection{Plant Material}

Wheat seeds of four varieties, Argine, and Elnilein (selected from varieties grown on salt-stressed soils in northern Sudan), Xumai 30, and Yang 10-13 (selected from varieties grown on salt-stressed soils in China) were used in this study. Seeds within a variety were selected for uniform size, shape, and color. All seeds were less than 18-mo old and were previously stored in paper bags under laboratory conditions (RH $40-60 \%$ at $15-20{ }^{\circ} \mathrm{C}$ ) to maintain good germination ability.

\subsection{Experimental Design}

The study was performed in a controlled environment in Jiangsu Provincial Key Lab of Crop Genetics and Physiology of Yangzhou University $\left(32^{\circ} 30^{\prime} \mathrm{N}, 119^{\circ} 25^{\prime} \mathrm{E}\right)$, Yangzhou, Jiangsu Province, China in the winter of 2016. The experimental design was a factorial design with three factors (four wheat varieties, three nitrogen levels and four salinity levels), arranged in a split plot randomized complete block design with three replications for each treatment.

Before sowing sand was washed and dried in $100{ }^{\circ} \mathrm{C}$. Plastic pots $(\mathrm{n}=72,9.5 \mathrm{~cm}$ in diameter by $8.5 \mathrm{~cm}$ in depth) were prepared, and each pot was filled with $400 \mathrm{~g}$ washed sand. Salinity was developed in each pot by supplying the first irrigation with the same amount $(80 \mathrm{~mL})$ of $0,100,150$ and $200 \mathrm{mM} \mathrm{NaCl}$ solution. Seeds were selected for uniform size, shape and color from each variety. Seeds were surface-sterilized with $1 \%$ sodium hypochlorite solution for 3 minutes then thoroughly rinsed three times with distilled water and air dried near to their original weight. Twenty seeds of each variety was sown in each pot. The plastic pots were placed in growth chambers (Model PYX-300G-B, Yangzhou Yiwei Automatic Instrument Co. Ltd, Jiangsu, China) for 21 days at $25^{\circ} \mathrm{C}$. The relative humidity was maintained at $60-70 \%$.

\subsection{Observations and Measurements}

\subsubsection{Emergence Percentage (EP)}

The number of emerged seedlings was recorded on a daily basis. Seedlings were considered emerged when coleoptiles were visible above the substratum surface. After ten days EP was calculated with the following formula:

$$
\mathrm{EP}=\text { Number of emerged seeds/Number of total seeds }) \times 100
$$

\subsubsection{Shoot and Root Length, Root Number}

At three weeks after sowing, five seedlings were sampled randomly and seedling growth was measured as shoot length, root length, and root number. 


\subsubsection{Total Dry Weight (TDW)}

The dry weight of five seedlings per pot was measured after drying shoot and root at $80{ }^{\circ} \mathrm{C}$ for 48 hours.

\subsubsection{Salt Tolerance Index (STI)}

Salt tolerance index was calculated as the ratio of the dry plant weight subjected to specific salt concentrations to the dry plant weight of the control $(0 \mathrm{mM} \mathrm{NaCl})$. Salt tolerance index was calculated according to (Hussien Ibrahim et al., 2016) as follows:

$$
\mathrm{STI}=\frac{\mathrm{TDW} \text { at } \mathrm{Sx}}{\mathrm{TDW} \text { at } \mathrm{S} 0} \times \frac{1}{100}
$$

$\mathrm{TDW}=$ Total dry $\mathrm{wt}, \mathrm{S} 0=$ control, $\mathrm{Sx}=\mathrm{a}$ given concentration out of salt concentrations.

\subsubsection{Seedling Vigour Index (SVI)}

Seedling vigour index (SVI) was calculated using the formula of Abdul-Baki and Anderson (1973), and Anderson (1973):

$$
\mathrm{SVI}=\frac{\text { Seedling Emergence Percentage } \times \text { Seedling Length }}{100}
$$

\subsection{Data Analysis}

The experimental design was a factorial experiment with three factors and arranged as a split plot in randomized complete block design with three replications for each treatment. The data of each variable were subjected to analysis of variance (ANOVA) with the statistical package of MSTATC (Version 2.00) according to this design. When $\mathrm{F}$ values were significant, means were separated by the LSD test $(\mathrm{P} \leq 0.05)$.

\section{Results}

The effects of variety (V), nitrogen (N), salinity (S), and their interactions are compiled in Table 1.

The results showed that seedling growth parameters of wheat varieties were significantly affected by $\mathrm{V}, \mathrm{S}, \mathrm{N}$ and their interactions on most occasions.

Table 1. Analysis of variance for effects of varieties (V), Nitrogen (N), Salt (S) and their interaction on seedling

\begin{tabular}{|c|c|c|c|c|c|c|c|}
\hline Parameter & Variety $(\mathrm{V})$ & Nitrogen $(\mathrm{N})$ & $\mathrm{V} \times \mathrm{N}$ & Salt (S) & $\mathrm{V} \times \mathrm{S}$ & $\mathrm{N} \times \mathrm{S}$ & $\mathrm{V} \times \mathrm{N} \times \mathrm{S}$ \\
\hline Emergence (\%) & $* *$ & $* *$ & $* *$ & $* *$ & $* *$ & $* *$ & $* *$ \\
\hline Shoot Length (cm) & $* *$ & ns & $* *$ & $* *$ & $* *$ & $* *$ & $\mathrm{~ns}$ \\
\hline Root Length(cm) & $* *$ & $* *$ & $* *$ & $* *$ & $* *$ & $* *$ & $* *$ \\
\hline Roots number & $*$ & $* *$ & ns & $* *$ & $\mathrm{~ns}$ & ns & $\mathrm{ns}$ \\
\hline Total Dry Weight (g) & $*$ & $* *$ & $* *$ & $* *$ & $* *$ & ns & $* *$ \\
\hline Salt Tolerance Index (STI) & ns & $* *$ & ns & $* *$ & $* *$ & ns & $* *$ \\
\hline Seedling Vigor Index (SVI) & $* *$ & ns & $* *$ & $* *$ & $* *$ & $* *$ & $*$ \\
\hline
\end{tabular}
growth parameters of wheat

Note. Ns $=$ Not significant difference; * Significant difference at $\mathrm{P} \leq 0.05 ; * *$ Significant difference at $\mathrm{P} \leq 0.01$.

\subsection{Emergence Percentage (EP)}

EP was significantly affected by varieties, nitrogen and salinity (Table1).

At different salt concentrations, and $\mathrm{N}$ treatments Elnilein had the highest EP (75.7\%), whereas Yang 10-13 had the lowest percentage (55.2\%). Compared to the control, the EP of seeds in the salinity treatments decreased with the increase of salinity level (Table 2). EP was decreased by 20.1, 35.7, and $49.9 \%$ in 100, 150, and $200 \mathrm{mM}$ $\mathrm{NaCl}$, respectively, compared with control. At $200 \mathrm{mM}, \mathrm{NaCl}$ Elnilein had better tolerance to salt stress than other varieties in terms of EP (Figure 1a, Table 2). In term of interaction between $\mathrm{N}$ and $\mathrm{S}$ results revealed that enhancement in $\mathrm{N}$ rates at high levels of $\mathrm{S}$ had positive effect on EP. Of the nitrogen levels, $2 \mathrm{~N}$ had the best promotional effects on seedling emergence (Figure 1b, Table 2). Argine had the highest percentage of emergence at $1 \mathrm{~N}$ and $2 \mathrm{~N}$ (Figure 1c). EP was increased at $200 \mathrm{mM} \mathrm{NaCl}$, and $2 \mathrm{~N}$ compared with the control (Figure 1). Elnilein had the highest EP of $65.67 \%$ at the $200 \mathrm{mM} \mathrm{NaCl}$ level, and $2 \mathrm{~N}$ (Table 2). 
Table 2. Effects of salinity and nitrogen levels on emergence percentage of four wheat varieties

\begin{tabular}{|c|c|c|c|c|c|}
\hline \multirow{2}{*}{ Nitrogen $(\mathrm{kg} / \mathrm{h})$} & \multirow{2}{*}{ Salinity (mM NaCl) } & \multicolumn{4}{|c|}{ Varieties } \\
\hline & & Argine & Elnilein & Xumai 30 & Yang 10-13 \\
\hline \multirow[t]{4}{*}{$0 \mathrm{~N}$} & 0 & $100 .^{\mathrm{a}}$ & $100.0^{\mathrm{a}}$ & $100.0^{\mathrm{a}}$ & $100.0^{\mathrm{a}}$ \\
\hline & 100 & $88.33^{\mathrm{c}}$ & $81.67^{\mathrm{fg}}$ & $79.33^{\text {gh }}$ & $65.00^{1 \mathrm{~m}}$ \\
\hline & 150 & $78.33^{\mathrm{h}}$ & $73.33^{\mathrm{ij}}$ & $74.33 \mathrm{ij}$ & $56.67^{\mathrm{op}}$ \\
\hline & 200 & $44.00^{\mathrm{s}}$ & $70.33^{\mathrm{k}}$ & $51.67^{\mathrm{q}}$ & $25.00^{\mathrm{t}}$ \\
\hline \multirow[t]{4}{*}{$1 \mathrm{~N}$} & 0 & $100.0^{\mathrm{a}}$ & $100.0^{\mathrm{a}}$ & $100.0^{\mathrm{a}}$ & $100.0^{\mathrm{a}}$ \\
\hline & 100 & $83.67^{\mathrm{ef}}$ & $91.00^{\mathrm{b}}$ & $85.33^{\mathrm{de}}$ & $58.33 \mathrm{o}$ \\
\hline & 150 & $73.33^{\mathrm{ij}}$ & $80.00^{\mathrm{gh}}$ & $63.33^{\mathrm{mn}}$ & $25.00^{\mathrm{t}}$ \\
\hline & 200 & $61.67^{\mathrm{n}}$ & $72.33^{\mathrm{jk}}$ & $50.00^{\mathrm{q}}$ & $15.00^{\mathrm{v}}$ \\
\hline \multirow[t]{4}{*}{$2 \mathrm{~N}$} & 0 & $100.0^{a}$ & $100.0^{a}$ & $100.0^{\mathrm{a}}$ & $100.0 \mathrm{a}$ \\
\hline & 100 & $85.00^{\mathrm{de}}$ & $93.33^{\mathrm{b}}$ & $86.33^{\mathrm{cd}}$ & $62.00^{\mathrm{n}}$ \\
\hline & 150 & $78.33^{h}$ & $46.67^{r}$ & $67.00^{1}$ & $55.00^{\mathrm{p}}$ \\
\hline & 200 & $65.67^{\mathrm{lm}}$ & $75.00^{\mathrm{i}}$ & $52.00^{\mathrm{q}}$ & $19.00^{\mathrm{u}}$ \\
\hline
\end{tabular}

Note. * Different letters at the same line and column show significant differences at 0.05 level.
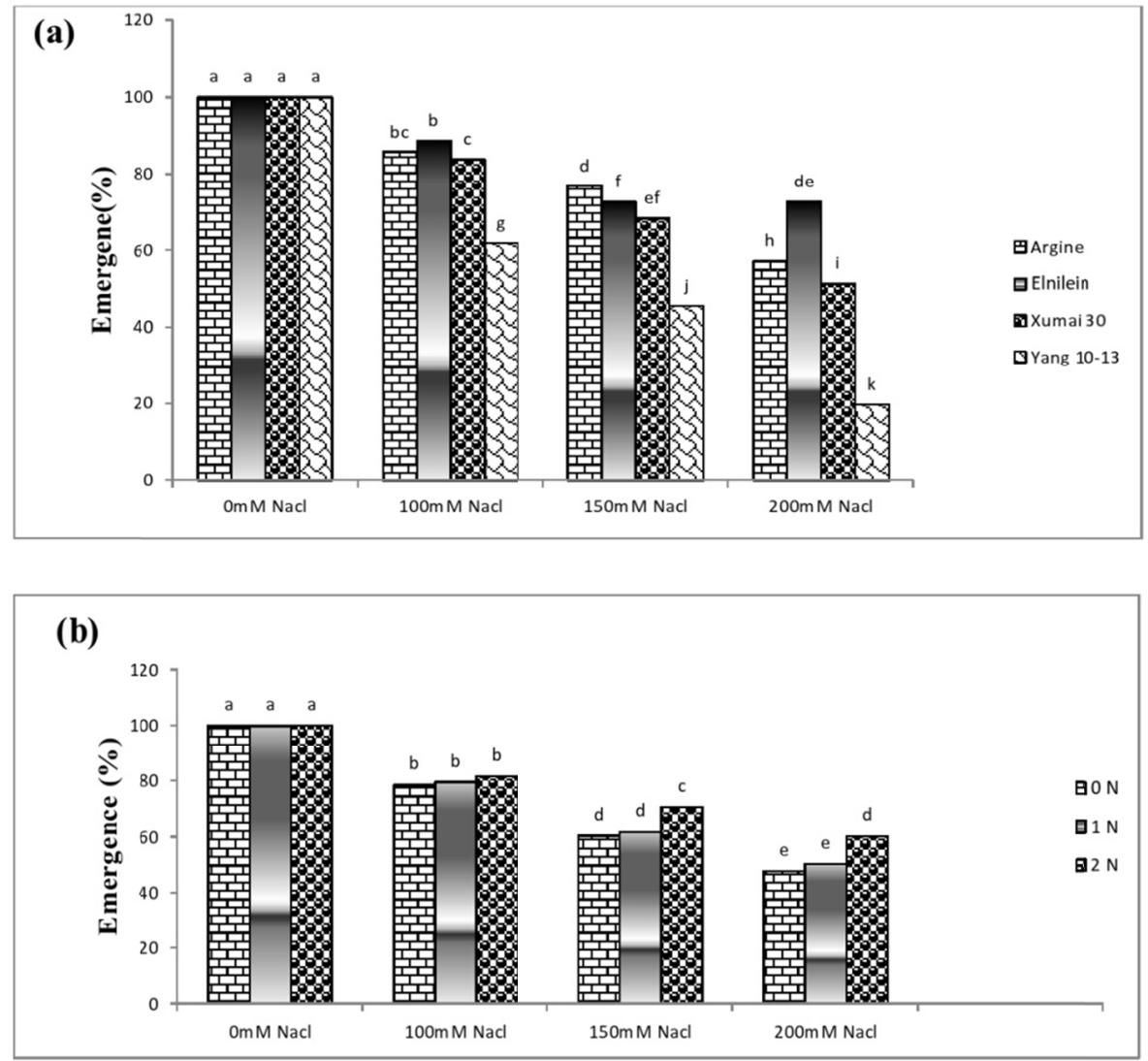


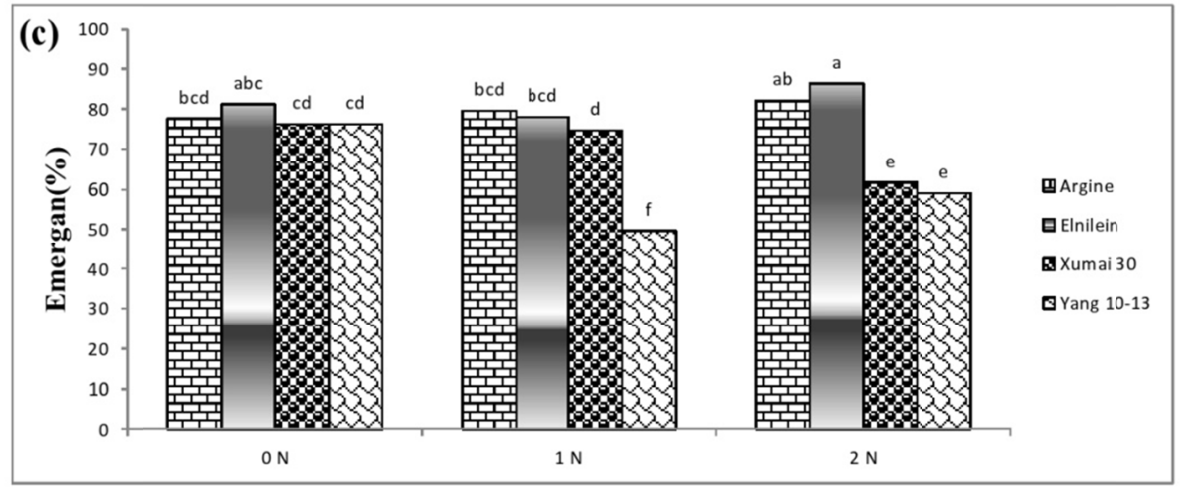

Figure 1. Effect on emergence percentage of four wheat varieties (Argine, Elnilein, Xumai 30, and Yang 10-13) of: (a) interaction between salinity and varieties-average calculated over nitrogen treatments; (b) interaction between salinity and nitrogen treatments—average calculated over varieties treatments; and (c) interaction between varieties and nitrogen treatments-average calculated over salinity treatments

Note. Bars different letters indicate significant differences at the 0.05 probability level. Means were separated by the LSD test if $\mathrm{F}$ values were significant.

\subsection{Growth Characteristics}

At high salinity level shoot and root increase with an increased $\mathrm{N}$ level (Figures $2 \mathrm{~b}$ and $3 \mathrm{~b}$ ). At the interaction between $\mathrm{V}$ and $\mathrm{N}$ levels Elnilein exhibited a greater shoot length than the other varieties at $\mathrm{N} 1$ and $2 \mathrm{~N}$ (Figure 2c). Root length was significantly affected by $\mathrm{N}$ levels. Furthermore, Elnilein had the longest root at all $\mathrm{N}$ levels (Figure 3c). This result showed that increment of $\mathrm{N}$ levels resulted in a significant reduction in salinity effect on shoot and root growth. At the $200 \mathrm{mM} \mathrm{NaCl}$ and $2 \mathrm{~N}$ levels, the longest and shortest shoot length was 19.86 and $12.70 \mathrm{~cm}$, respectively, measured in Elnilein and Yang 10-13 (Table 3), whereas the longest and shortest root length was 8.10 and $4.4 \mathrm{~cm}$ measured in Elnilein and Yang 10-13 at $200 \mathrm{mM} \mathrm{NaCl}$, and $2 \mathrm{~N}$ respectively (Table 4).

Table 3. Effects of different salinity and nitrogen levels on seedling shoot length of four wheat varieties

\begin{tabular}{|c|c|c|c|c|c|}
\hline \multirow{2}{*}{ Nitrogen $(\mathrm{kg} / \mathrm{h})$} & \multirow{2}{*}{ Salinity $(\mathrm{mM} \mathrm{NaCl})$} & \multicolumn{4}{|c|}{ Varieties } \\
\hline & & Argine & Elnilein & Xumai 30 & Yang $10-13$ \\
\hline \multirow[t]{4}{*}{$0 \mathrm{~N}$} & 0 & $48 \mathrm{i}^{\mathrm{jk}}$ & $27.85^{\mathrm{ij}}$ & $29.21^{\mathrm{gh}}$ & $27.44^{\mathrm{jk}}$ \\
\hline & 100 & $25.13^{\text {no }}$ & $26.23^{\mathrm{lm}}$ & $25.27^{\mathrm{mno}}$ & $25.88^{\operatorname{lmn}}$ \\
\hline & 150 & $22.08 \mathrm{~s}^{\mathrm{t}}$ & $22.20^{\mathrm{s}}$ & $23.22^{\mathrm{qr}}$ & $22.65^{\mathrm{rs}}$ \\
\hline & 200 & $15.6^{8 y}$ & $17.45^{\mathrm{wxkl}}$ & $21.21^{\mathrm{tu}}$ & $15.09^{y}$ \\
\hline \multirow[t]{4}{*}{$1 \mathrm{~N}$} & 0 & $29.57^{\mathrm{fg}}$ & $31.88^{\mathrm{cd}}$ & $31.13^{\mathrm{de}}$ & $30.27^{\mathrm{e} \tilde{\mathrm{f}}}$ \\
\hline & 100 & $26.34^{1}$ & $27.55^{\mathrm{ijk}}$ & $26.61^{\mathrm{kl}}$ & $27.62^{\mathrm{ij}}$ \\
\hline & 150 & $22.60^{\mathrm{rs}}$ & $25.76^{\mathrm{lmn}}$ & $21.85^{\mathrm{st}}$ & $16.70^{\mathrm{x}}$ \\
\hline & 200 & $17.30^{\mathrm{wx}}$ & $20.20^{\mathrm{v}}$ & $17.00^{\mathrm{wx}}$ & $7.200^{[}$ \\
\hline \multirow[t]{5}{*}{$2 \mathrm{~N}$} & 0 & $33.63^{b^{--}}$ & $35.37^{\mathrm{a}}$ & $32.27^{\mathrm{c}}$ & $32.77^{\mathrm{bc}-}$ \\
\hline & 100 & $26.07^{1 \mathrm{mn}}$ & $28.45^{\mathrm{hi}}$ & $24.53^{\mathrm{op}}$ & $23.72^{\mathrm{pq}}$ \\
\hline & 150 & $21.75^{\mathrm{st}}$ & $23.29^{\mathrm{qr}}$ & $20.73^{\mathrm{uv}}$ & $20.00^{\mathrm{v}}$ \\
\hline & 200 & $17.89^{\mathrm{w}}$ & $19.86^{\mathrm{v}}$ & $15.67^{\mathrm{y}}$ & $12.70^{\mathrm{z}}$ \\
\hline & $\mathrm{CV} \%$ & $10.10 \%$ & & & \\
\hline
\end{tabular}

Note. * Different letters at the same line and column show significant differences at 0.05 level. 

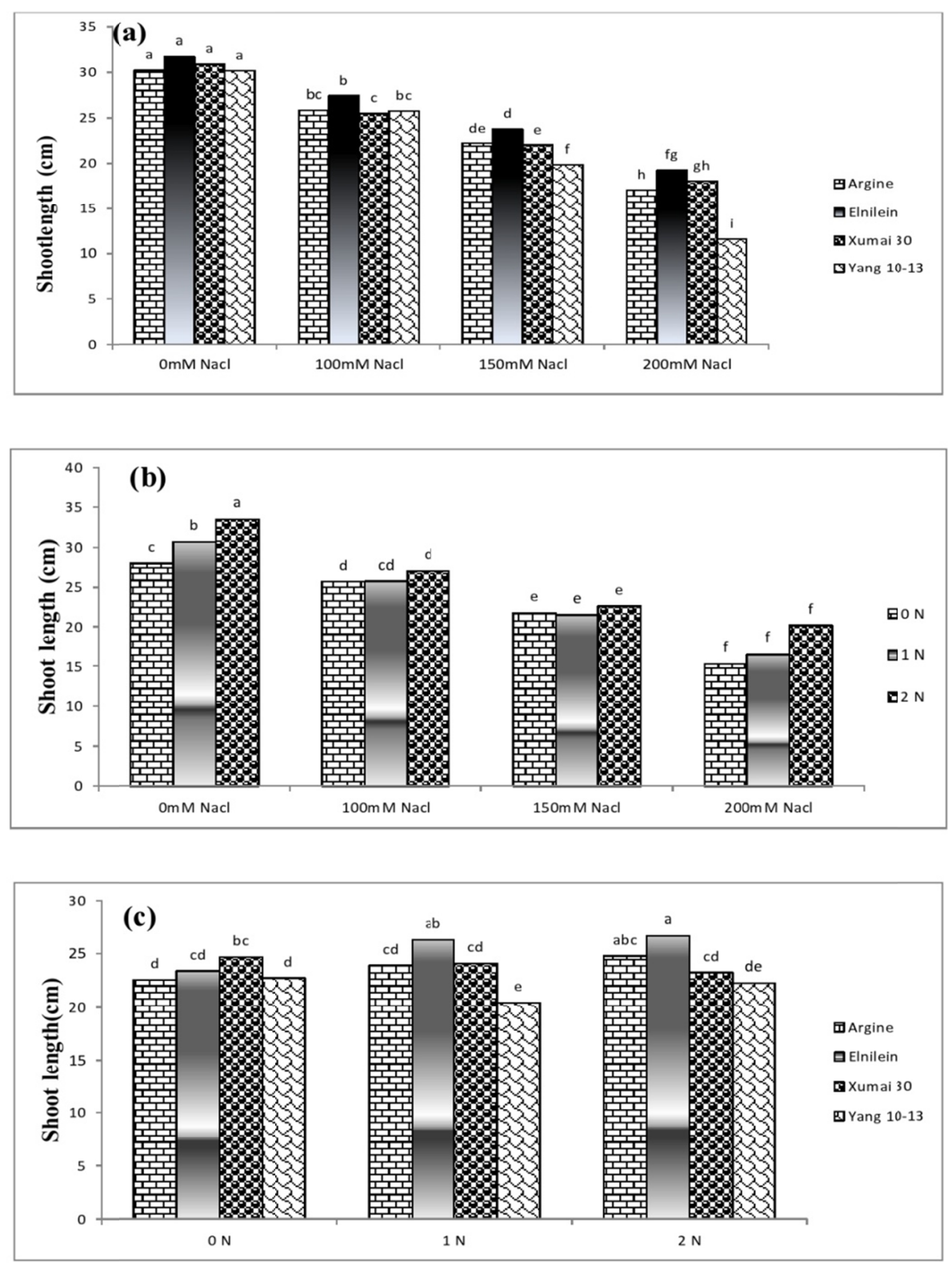

Figure 2. Effect on shoot length of four wheat varieties (Argine, Elnilein, Xumai 30, and Yang 10-13) of: (a) interaction between salinity and varieties - average calculated over nitrogen treatments; (b) interaction between salinity and nitrogen treatments - average calculated over varieties treatments; and (c) interaction between varieties and nitrogen treatments - average calculated over salinity treatments

Note. Bars different letters indicate significant differences at the 0.05 probability level. Means were separated by the LSD test if $\mathrm{F}$ values were significant. 

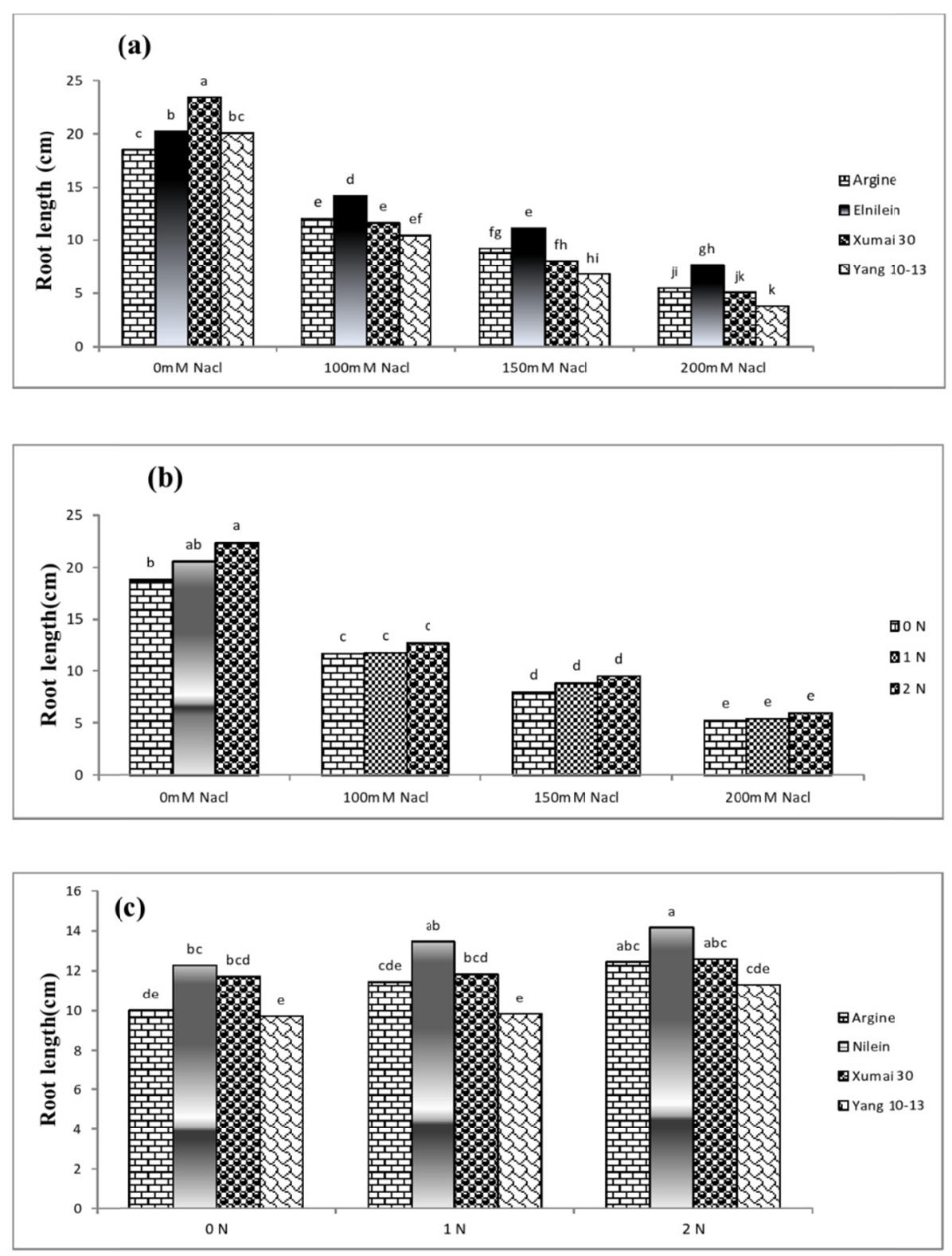

Figure 3. Effect on root length of four wheat varieties (Argine, Elnilein, Xumai 30, and Yang 10-13) of: (a) interaction between salinity and varieties - average calculated over nitrogen treatments; (b) interaction between salinity and nitrogen treatments - average calculated over varieties treatments; and (c) interaction between varieties and nitrogen treatments - average calculated over salinity treatments

Note. Bars different letters indicate significant differences at the 0.05 probability level. Means were separated by the LSD test if $\mathrm{F}$ values were significant.

The results showed that root number of wheat varieties was significantly affected by $\mathrm{V}, \mathrm{N}$, and $\mathrm{S}$. But there were no significant differences on interaction among experimental factors (Table 1). Root number increased with increased salinity levels. Compared with $0 \mathrm{mM} \mathrm{NaCl}, 100 \mathrm{mM} \mathrm{NaCl}$ increased root number by $33.7 \%$ (Figure 4). The highest root number was 8.53 measured in Elnilein at $200 \mathrm{mM} \mathrm{NaCl}$ and $2 \mathrm{~N}$ (Table5). 
Table 4. Effects of different salinity and nitrogen levels as $(0,105$ and $210 \mathrm{~kg} \mathrm{~N} / \mathrm{h}$, designated as $0 \mathrm{~N}, 1 \mathrm{~N}$, and $2 \mathrm{~N}$ respectively) concentrations on root length of four wheat varieties

\begin{tabular}{|c|c|c|c|c|c|}
\hline \multirow{2}{*}{ Nitrogen $(\mathrm{kg} / \mathrm{h})$} & \multirow{2}{*}{ Salinity (mM NaCl) } & \multicolumn{4}{|c|}{ Varieties } \\
\hline & & Argine & Elnilein & Xumai 30 & Yang $10-13$ \\
\hline \multirow[t]{4}{*}{$0 \mathrm{~N}$} & 0 & $17.14^{\mathrm{h}}$ & $18.00^{\mathrm{gh}}$ & $21.55^{\mathrm{c}}$ & $18.43^{\mathrm{fg}}$ \\
\hline & 100 & $10.77^{\text {opqr }}$ & $13.43^{\mathrm{jk}}$ & $12.68^{\mathrm{kl}}$ & $9.913^{\mathrm{rs}}$ \\
\hline & 150 & $7.287^{\mathrm{vwx}}$ & $10.63^{\mathrm{opqr}}$ & $7.353^{\mathrm{vwx}}$ & $6.867^{\mathrm{wxy}}$ \\
\hline & 200 & $4.807^{\complement \backslash}$ & $7.080^{\mathrm{wx}}$ & $5.300^{z[\backslash}$ & $3.600^{1^{\wedge}}$ \\
\hline \multirow[t]{4}{*}{$1 \mathrm{~N}$} & 0 & $18.57^{\mathrm{fg}^{-}}$ & $20.37^{\mathrm{d}^{-}}$ & $24.10^{\mathrm{a}}$ & $19.28^{\mathrm{ef}}$ \\
\hline & 100 & $12.13^{\mathrm{lm}}$ & $14.73^{\mathrm{i}}$ & $10.23^{\mathrm{pqrs}}$ & $10.03^{\mathrm{qrs}}$ \\
\hline & 150 & $9.443^{\mathrm{st}}$ & $11.00^{\text {nop }}$ & $8.317^{\mathrm{u}}$ & $6.667 x y$ \\
\hline & 200 & $5.650^{z[}$ & $7.767^{\mathrm{uvw}}$ & $4.700^{`}$ & $3.333^{\wedge}$ \\
\hline \multirow[t]{5}{*}{$2 \mathrm{~N}$} & 0 & $19.77 \mathrm{de}$ & $22.40^{\mathrm{bc}-}$ & $24.77^{\mathrm{a}}$ & $22.60^{\mathrm{b}}$ \\
\hline & 100 & $13.13^{\mathrm{k}}$ & $14.32^{\mathrm{ij}}$ & $11.96^{\mathrm{lm}}$ & $11.37^{\mathrm{mno}}$ \\
\hline & 150 & $10.93^{\text {nopq }}$ & $11.85^{\operatorname{lmn}}$ & $8.533^{\text {tu }}$ & $6.900^{w x y}$ \\
\hline & 200 & $6.000^{\mathrm{yz}}$ & $8.100^{\mathrm{uv}}$ & $5.180^{z\lceil\backslash}$ & $4.433^{\backslash]}$ \\
\hline & $\mathrm{CV} \%$ & $19.60 \%$ & & & \\
\hline
\end{tabular}

Note. * Different letters at the same line and column show significant differences at 0.05 level.

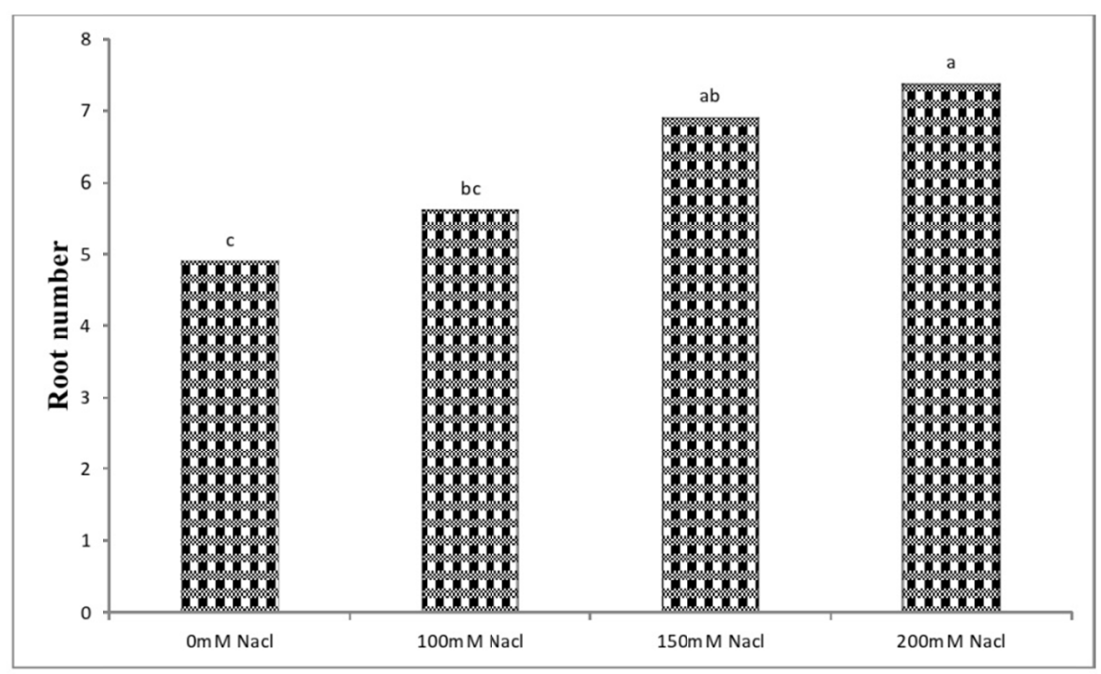

Figure 4. Effects of salinity $(\mathrm{NaCl})$ on root number based on data from Table 5 (mean of the means for each salinity level)

Note. Different letters indicate significant differences at the 0.05 probability level. Means were separated by the LSD test if $\mathrm{F}$ values were significant. 

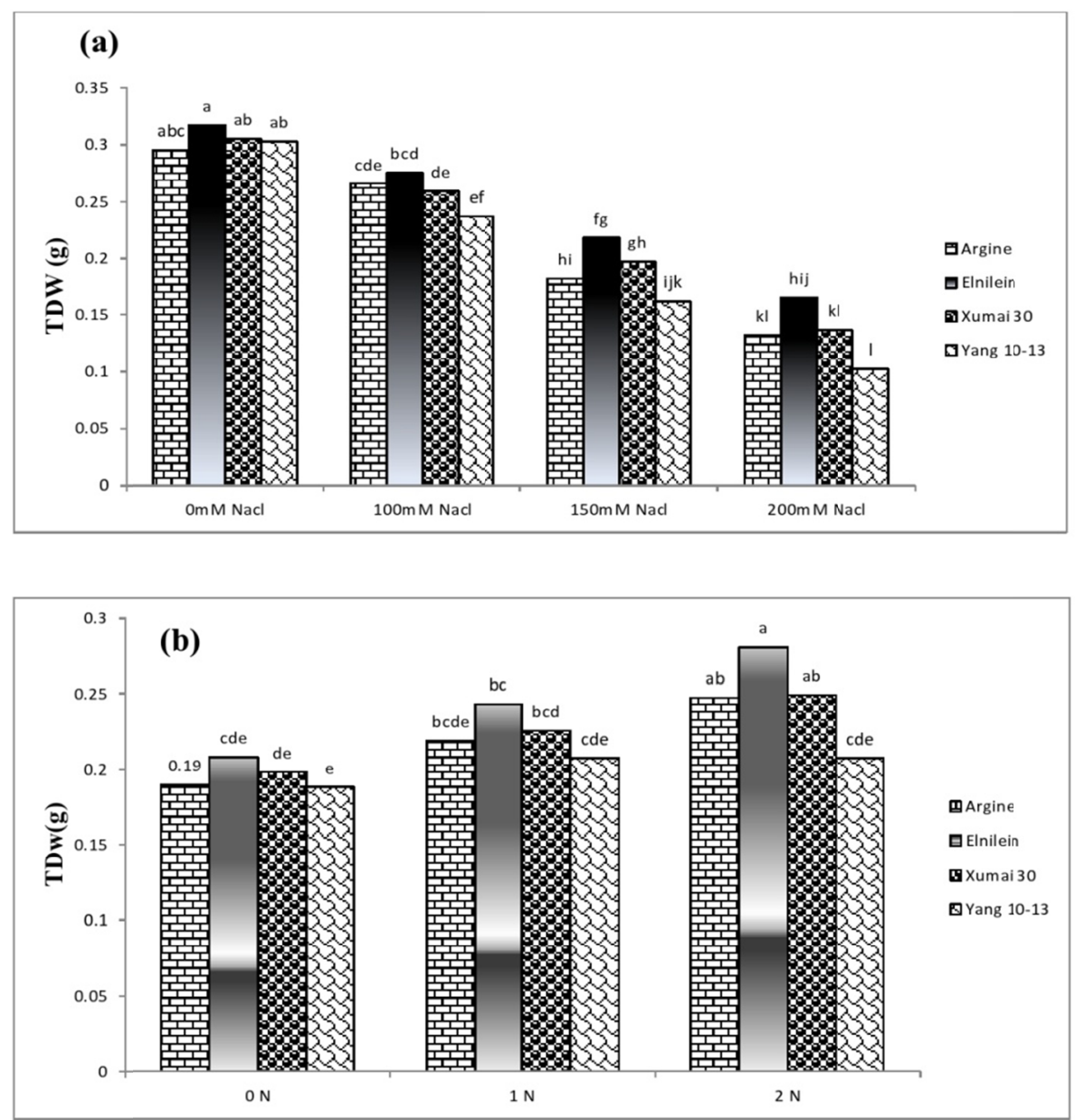

Figure 5. Effect on dry weight of four wheat varieties (Argine, Elnilein, Xumai 30, and Yang 10-13) of: (a) interaction between salinity and varieties - average calculated over nitrogen treatments; (b) interaction between varieties and nitrogen treatments - average calculated over salinity treatments

Note. Bars different letters indicate significant differences at the 0.05 probability level. Means were separated by the LSD test if $\mathrm{F}$ values were significant.

Total dry weight was significantly affected by V, N, S and their combinations except the interaction between $\mathrm{N}$ and $\mathrm{S}$ levels (Table 1).

The total dry weight was negatively affected by increasing salinity level (Table 6). The average total dry weight of the four varieties was $0.31 \mathrm{~g}$ in the control $(0 \mathrm{Mm})$ and this value gradually decreased to $.13 \mathrm{~g}$ at the $200 \mathrm{mM}$ $\mathrm{NaCl}$ level. Compared with the control, plant dry weight was significantly reduced at the $200 \mathrm{mM} \mathrm{NaCl}$ level, with a reduction of $55.28 \%$ for Argine, $47.89 \%$ for Elnilein, $55.27 \%$ for Xumai and $66.30 \%$ for Yang $10-13$ (Figure 5a).

The results also showed that the total dry weight was positively affected by increasing $\mathrm{N}$ level (Table 6). Compared with the control $(0 \mathrm{~N})$, dry plant weight was significantly enhanced at the $2 \mathrm{~N}$ level, with an increase of 23.23\% for Argine, $25.82 \%$ for Elnilein, $20.43 \%$ for Xumai 30, and 9.25\% for Yang 10-13 (Figure 5b). 
Table 5. Effects of different salinity and nitrogen levels on number of root of four wheat varieties

\begin{tabular}{|c|c|c|c|c|c|}
\hline \multirow{2}{*}{ Nitrogen $(\mathrm{kg} / \mathrm{h})$} & \multirow{2}{*}{ Salinity $(\mathrm{mM} \mathrm{NaCl})$} & \multicolumn{4}{|c|}{ Varieties } \\
\hline & & Argine & Elnilein & Xumai 30 & Yang 10-13 \\
\hline \multirow[t]{4}{*}{$0 \mathrm{~N}$} & 0 & $4.900^{\circ}$ & $5.467^{\mathrm{m}}$ & $5.667^{\mathrm{lm}}$ & $5.733^{\mathrm{klm}}$ \\
\hline & 100 & $6.167^{\mathrm{ij}}$ & $5.667^{\mathrm{lm}}$ & $5.733^{\mathrm{klm}}$ & $6.267^{\mathrm{ij}}$ \\
\hline & 150 & $7.400^{\mathrm{ef}}$ & $7.133^{\mathrm{fg}}$ & $6.800^{\mathrm{gh}}$ & $7.000^{\mathrm{fg}}$ \\
\hline & 200 & $7.067^{\mathrm{fg}}$ & $8.233^{\mathrm{ab}}$ & $7.100^{\mathrm{fg}}$ & $7.133^{\mathrm{fg}}$ \\
\hline \multirow[t]{4}{*}{$1 \mathrm{~N}$} & 0 & $4.867^{\circ}$ & $4.667^{\circ}$ & $5.000^{\mathrm{no}}$ & $4.667^{\circ}$ \\
\hline & 100 & $6.467^{\mathrm{hi}}$ & $5.000^{\text {no }}$ & $5.467^{\mathrm{m}}$ & $5.667^{\mathrm{lm}}$ \\
\hline & 150 & $7.100^{\mathrm{fg}}$ & $7.600^{\mathrm{de}}$ & $7.400^{\text {ef }}$ & $7.133^{\mathrm{fg}}$ \\
\hline & 200 & $8.033^{\mathrm{bc}}$ & $8.530^{\mathrm{a}}$ & $7.567^{\mathrm{e}}$ & $5.000^{\mathrm{no}}$ \\
\hline \multirow[t]{5}{*}{$2 \mathrm{~N}$} & 0 & $4.667^{\circ}$ & $4.900^{\circ}$ & $3.467^{\mathrm{p}}$ & $4.667^{\circ}$ \\
\hline & 100 & $5.333^{\mathrm{mn}}$ & $5.000^{\text {no }}$ & $5.333^{\mathrm{mn}}$ & $5.333^{\mathrm{mn}}$ \\
\hline & 150 & $7.667^{\text {cde }}$ & $6.133^{\mathrm{ijk}}$ & $5.467^{\mathrm{m}}$ & $6.000^{\mathrm{jkl}}$ \\
\hline & 200 & $7.633^{\text {cde }}$ & $8.000^{\mathrm{bcd}}$ & $6.000^{\mathrm{jkl}}$ & $8.133^{\mathrm{ab}}$ \\
\hline & $\mathrm{CV} \%$ & $15.50 \%$ & & & \\
\hline
\end{tabular}

Note. ${ }^{*}$ Different letters at the same line and column show significant differences at 0.05 level.

Table 6. Effects of different salinity and nitrogen levels on TDw of four wheat varieties

\begin{tabular}{|c|c|c|c|c|c|}
\hline \multirow{2}{*}{ Nitrogen $(\mathrm{kg} / \mathrm{h})$} & \multirow{2}{*}{ Salinity (mM NaCl) } & \multicolumn{4}{|c|}{ Varieties } \\
\hline & & Argine & Elnilein & Xumai 30 & Yang 10-13 \\
\hline \multirow[t]{4}{*}{$0 \mathrm{~N}$} & 0 & $0.2567^{\mathrm{hij}}$ & $0.2567^{\text {hij }}$ & $0.2767^{\mathrm{fg}}$ & $0.3100^{\mathrm{cd}}$ \\
\hline & 100 & $0.2333^{\mathrm{klm}}$ & $0.2367^{\mathrm{kl}}$ & $0.2067^{\mathrm{op}}$ & $0.2167^{\mathrm{mno}}$ \\
\hline & 150 & $0.1500^{\mathrm{st}}$ & $0.1900^{\mathrm{pq}}$ & $0.1733^{\mathrm{qr}}$ & $0.1333^{\text {tuv }}$ \\
\hline & 200 & $0.1200^{\mathrm{uvw}}$ & $0.1500^{\mathrm{st}}$ & $0.1367^{\mathrm{tu}}$ & $0.09333^{\mathrm{x}}$ \\
\hline \multirow[t]{4}{*}{$1 \mathrm{~N}$} & 0 & $0.2967^{\mathrm{de}}$ & $0.3233^{\mathrm{bc}}$ & $0.3067^{\text {de }}$ & $0.2900^{\mathrm{ef}}$ \\
\hline & 100 & $0.2733^{\text {fgh }}$ & $0.2767^{\mathrm{fg}}$ & $0.2967^{\mathrm{de}}$ & $0.2667^{\text {ghi }}$ \\
\hline & 150 & $0.1900^{\mathrm{pq}}$ & $0.2133^{\text {no }}$ & $0.1767^{\mathrm{qr}}$ & $0.1667^{\mathrm{rs}}$ \\
\hline & 200 & $0.1167^{\mathrm{vw}}$ & $0.1600^{\mathrm{rs}}$ & $0.1233^{\mathrm{uvw}}$ & $0.1067^{\mathrm{wx}}$ \\
\hline \multirow[t]{5}{*}{$2 \mathrm{~N}$} & 0 & $0.3333^{\mathrm{b}}$ & $0.3733^{\mathrm{a}}$ & $0.3333^{\mathrm{b}}$ & $0.3100^{\mathrm{cd}^{-}}$ \\
\hline & 100 & $0.2900^{\mathrm{ef}}$ & $0.3133^{\mathrm{cd}}$ & $0.2733^{\mathrm{fgh}}$ & $0.2267^{\mathrm{lmn}}$ \\
\hline & 150 & $0.2067^{\mathrm{op}}$ & $0.2500^{\mathrm{ijk}}$ & $0.2400^{\mathrm{jkl}}$ & $0.1867^{\mathrm{q}}$ \\
\hline & 200 & $0.1600^{\mathrm{rs}}$ & $0.1867^{\mathrm{q}}$ & $0.1500^{\mathrm{st}}$ & $0.1067^{\mathrm{wx}}$ \\
\hline & $\mathrm{CV} \%$ & $18.20 \%$ & & & \\
\hline
\end{tabular}

Note. * Different letters at the same line and column show significant differences at 0.05 level.

\subsection{Salt Tolerance Index (STI)}

Salt tolerance at the early seedling stage, expressed with salt tolerance index, varied among the varieties. On average, Elnilein had the highest STI whereas Yang 10-13 had the lowest (Table 7). Argine demonstrated a better tolerance to salt stress than other varieties at $150 \mathrm{mM} \mathrm{NaCl}$. However, at the $200 \mathrm{mM} \mathrm{NaCl}$, Elnilein had the highest STI (Figure 6a). Based on STI at seedling growth stage, the varieties followed a descending STI of Elnilein, Argine Xumai 30, and Yang 10-13 (Table 7). Nitrogen rates affected the STI; likewise, interactions between salinity and nitrogen were significant (Table 1). In term of interaction between salinity and nitrogen levels, results showed that at $200 \mathrm{mM} \mathrm{NaCl} 2 \mathrm{~N}$ had the highest salt tolerance index. Results revealed that $\mathrm{N}$ had a positive effect on the STI at the high salinity level (Figure 6b). 
Table 7. Effects of different salinity and nitrogen on salt tolerance index (STI) of four wheat varieties

\begin{tabular}{|c|c|c|c|c|c|}
\hline \multirow{2}{*}{ Nitrogen $(\mathrm{kg} / \mathrm{h})$} & \multirow{2}{*}{ Salinity $(\mathrm{mM} \mathrm{NaCl})$} & \multicolumn{4}{|c|}{ Varieties } \\
\hline & & Argine & Elnilein & Xumai 30 & Yang 10-13 \\
\hline \multirow[t]{4}{*}{$0 \mathrm{~N}$} & 0 & $100.0^{\mathrm{ij}}$ & $100.0^{\mathrm{ij}}$ & $100.0 \mathrm{ij}$ & $100.0^{\mathrm{ij}}$ \\
\hline & 100 & $93.88^{\mathrm{j}}$ & $94.08^{\mathrm{j}}$ & $73.62^{\mathrm{lm}}$ & $70.24^{\mathrm{mn}}$ \\
\hline & 150 & $59.01^{\mathrm{pq}}$ & $75.63^{\operatorname{lm}}$ & $63.53^{\mathrm{op}}$ & $43.41^{\mathrm{t}}$ \\
\hline & 200 & $48.21^{\mathrm{st}}$ & $59.74^{\mathrm{pq}}$ & $51.30^{\mathrm{rs}}$ & $30.40^{\mathrm{u}}$ \\
\hline \multirow[t]{4}{*}{$1 \mathrm{~N}$} & 0 & $120.1^{\mathrm{de}}$ & $127.8^{\mathrm{bc}}$ & $112.4^{\mathrm{fg}^{-}}$ & $96.19^{i j}$ \\
\hline & 100 & $110.0^{\mathrm{g}}$ & $110.5 \mathrm{~g}$ & $106.7^{\mathrm{gh}}$ & $87.06^{\mathrm{k}}$ \\
\hline & 150 & $76.54^{1}$ & $85.3519^{\mathrm{ij}}$ & $65.99^{\text {no }}$ & $54.52^{\mathrm{qr}}$ \\
\hline & 200 & $45.04^{\mathrm{t}}$ & $62.56^{\mathrm{op}}$ & $44.83^{\mathrm{t}}$ & $33.33^{\mathrm{u}}$ \\
\hline \multirow[t]{5}{*}{$2 \mathrm{~N}$} & 0 & $134.0^{\mathrm{b}}$ & $148.1^{-\mathrm{a}}$ & $117.1^{\mathrm{ef}^{-}}$ & $100.9^{\mathrm{h}^{-}}$ \\
\hline & 100 & $120.2^{\mathrm{de}}$ & $124.8^{\mathrm{cd}}$ & $96.41^{\mathrm{ij}}$ & $72.94^{1 \mathrm{~m}}$ \\
\hline & 150 & $85.09^{\mathrm{k}}$ & $99.51^{\mathrm{ij}}$ & $84.10^{\mathrm{k}}$ & $59.50^{\mathrm{pq}}$ \\
\hline & 200 & $62.41^{\mathrm{op}}$ & $74.48^{\mathrm{lm}}$ & $52.56^{\mathrm{rs}}$ & $34.84^{\mathrm{u}}$ \\
\hline & $\mathrm{CV} \%$ & 18.51 & & & \\
\hline
\end{tabular}

Note. ${ }^{*}$ Different letters at the same line and column show significant differences at 0.05 level.
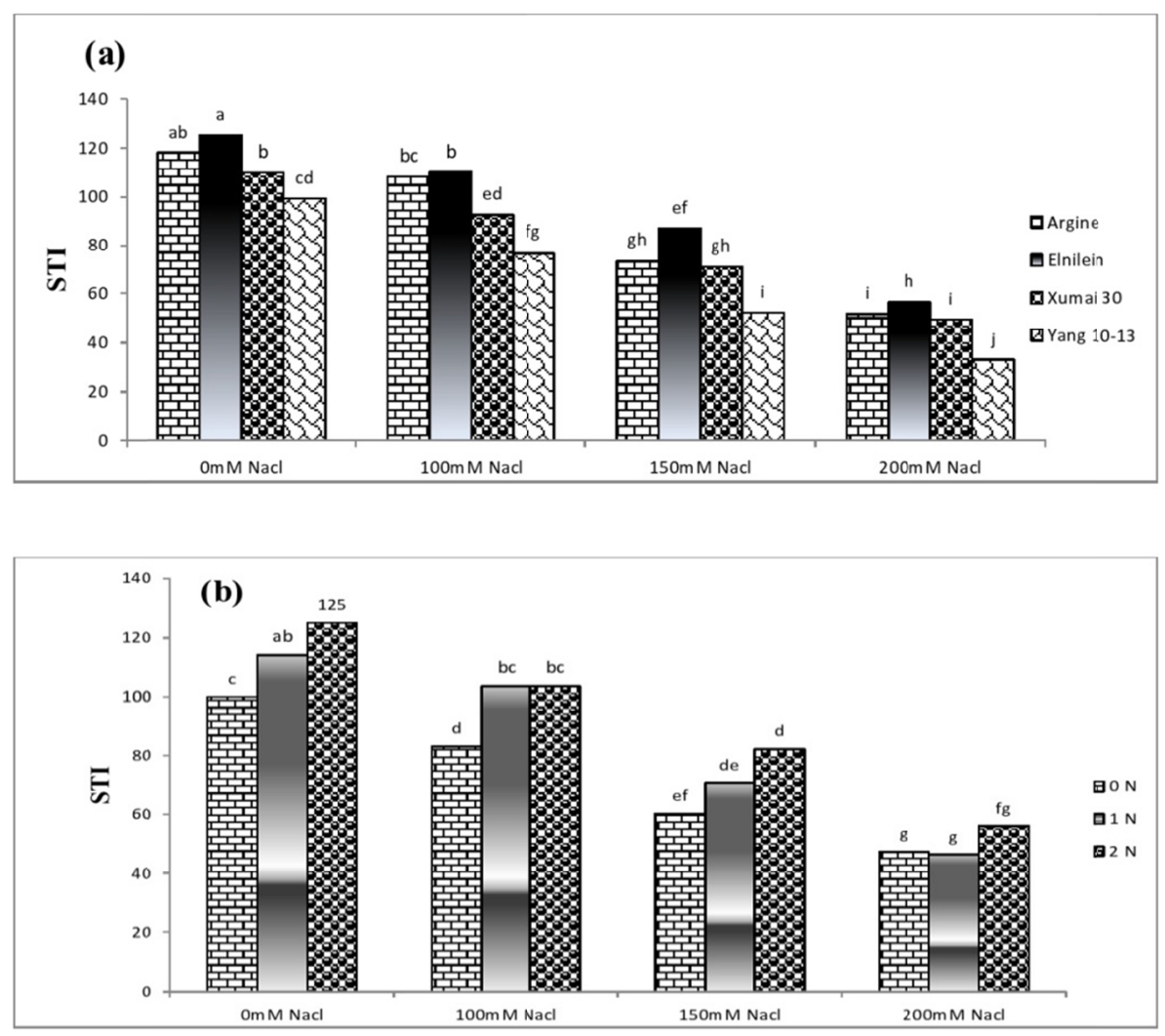

Figure 6. Effect on STI of four wheat varieties (Argine, Elnilein, Xumai 30, and Yang 10-13) of: (a) interaction between salinity and varieties_ _ average calculated over nitrogen treatments; (b) interaction between salinity and nitrogen treatments - average calculated over varieties treatments

Note. Bars different letters indicate significant differences at the 0.05 probability level. Means were separated by the LSD test if $\mathrm{F}$ values were significant. 


\subsection{Seedling Vigor Index (SVI)}

Results showed that SVI was significantly affected by all experimental factors and their combinations except nitrogen levels (Table 1). SVI decreased with increased salinity levels, likewise, increased with increased nitrogen levels (Table 8). On average, according to SVI, the varieties as the result of mean of the means were arranged as Elnilein (21.48), Argine (19.75), Xumai 30(19.10), and Yang 10-13(14.47) (Table 8).

Table 8. Effects of different salinity and nitrogen levels on SVI of four wheat varieties

\begin{tabular}{|c|c|c|c|c|c|}
\hline \multirow{2}{*}{ Nitrogen $(\mathrm{kg} / \mathrm{h})$} & \multirow{2}{*}{ Salinity (mM NaCl) } & \multicolumn{4}{|c|}{ Varieties } \\
\hline & & Argine & Elnilein & Xumai 30 & Yang 10-13 \\
\hline \multirow[t]{4}{*}{$0 \mathrm{~N}$} & 0 & $27.47^{\mathrm{hi}}$ & $27.83^{\mathrm{h}}$ & $29.20^{\mathrm{g}}$ & $27.47^{\mathrm{hi}}$ \\
\hline & 100 & $22.13^{\mathrm{kl}}$ & $21.50^{\operatorname{lm}}$ & $20.23^{\mathrm{n}}$ & $16.87^{\mathrm{opq}}$ \\
\hline & 150 & $17.33^{\circ}$ & $16.30^{\mathrm{pqr}}$ & $17.23^{\mathrm{op}}$ & $12.80^{\mathrm{u}}$ \\
\hline & 200 & $6.767^{z}$ & $12.30^{\mathrm{uv}}$ & $10.80^{\mathrm{wx}}$ & $3.800^{[}$ \\
\hline \multirow[t]{4}{*}{$1 \mathrm{~N}$} & 0 & $29.57^{\mathrm{fg}}$ & $31.87^{\mathrm{cd}^{-}}$ & $31.13^{\mathrm{de}}$ & $30.27^{\mathrm{ef}}$ \\
\hline & 100 & $22.00^{\mathrm{kl}}$ & $25.00^{\mathrm{j}}$ & $22.73^{\mathrm{k}}$ & $16.20^{\mathrm{qr}}$ \\
\hline & 150 & $16.60^{\text {opq }}$ & $20.60^{\mathrm{mn}}$ & $13.93^{\mathrm{t}}$ & $4.200^{[}$ \\
\hline & 200 & $10.67^{x}$ & $15.30^{\mathrm{rs}}$ & $8.500^{y}$ & $1.100^{]}$ \\
\hline \multirow[t]{5}{*}{$2 \mathrm{~N}$} & 0 & $33.63^{b^{-}}$ & $35.37^{\mathrm{a}}$ & $32.27^{\mathrm{c}}$ & $32.77^{\mathrm{bc}-}$ \\
\hline & 100 & $22.13^{\mathrm{kl}}$ & $26.50^{\mathrm{i}}$ & $21.17^{1 \mathrm{mn}}$ & $14.70^{\mathrm{st}}$ \\
\hline & 150 & $17.03^{\mathrm{opq}}$ & $10.90^{\mathrm{wx}}$ & $13.83^{\mathrm{t}}$ & $11.00^{\mathrm{wx}}$ \\
\hline & 200 & $11.73^{\mathrm{Vw}}$ & $14.33^{\mathrm{st}}$ & $8.133^{\mathrm{y}}$ & $2.400 \backslash$ \\
\hline & $\mathrm{CV} \%$ & $13.37 \%$ & & & \\
\hline
\end{tabular}

* Different letters at the same line and column show significant differences at 0.05 level.

\section{Discussions}

Crop establishment is critical for achieving reasonable economic yield. High-quality germination and good early seedling growth are critical for crop establishment. The vigorous growth of seedlings, often expressed with root and shoots growth, usually lays a solid foundation for an optimum plant population, especially under saline soil conditions (Zhou et al., 2010). Four wheat varieties from two different salt-affected regions in China and Sudan showed similar trends to $\mathrm{N}$ applications, and salinity stress in this experiment, but there were varietal differences in degree of reduction in seedling emergence, and early seedling growth. These differences suggest that a seedling growth stage could help in selection of suitable wheat varieties for salt-affected soils.

In this study, a slight decrease in seedling emergence was observed by increasing salinity levels in the investigated varieties (Table 2). This is in agreement with previous reports in sweet sorghum (Sorghum bicolor $\mathrm{L}$. Moench) (Nimir et al., 2014) and in wheat (Hussien Ibrahim et al., 2016). The research results were similar to the reports of many researchers (Kochak-Zadeh et al., 2013; Abdoli et al., 2013; Akbarimoghaddam et al., 2011; Rahman et al., 2008; Atak et al., 2006; Hampson \& Simpson, 1990; Neumann, 1995). They determined that seed germination and seedling growth reduced in saline soils with varying responses for varieties. The decline in EP under salinity has been attributed to the combined effect of osmotic pressure (Moud \& Maghsoudi, 2008) and toxicity of salts (Saboora et al., 2006) or due to the effect of added chlorine ion (Almodares et al., 2007) that gave rise to osmotic stress. Rahman et al. (2008) reported that the delay in germination by salinity mainly resulted from the altered water relations caused by high salt accumulation in the intercellular spaces. Furthermore, our results indicated that nitrogen accelerated the EP (Table 2). Similar results were obtained with Xiong et al. (2013). In the present study, the lowest emergence percentage was observed at the $200 \mathrm{mM} \mathrm{NaCl}$ with the $1 \mathrm{~N}$ level (Tables 2). However, in term of emergence percentage the affect of interaction, between $\mathrm{S}$ and $\mathrm{N}$ was significant, as with increase of $\mathrm{N}$ level, the EP increased at high levels of salinity (Figure 1b).

There are not a lot of studies on the effects of $\mathrm{N}$ on the early seedling growth of plants under saline conditions, but $\mathrm{N}$ containing compound accumulation is usually correlated with plant salt tolerance, even though this correlation is based on untested hypotheses (Mansour, 2000). Results of Al-Harbi et al. (2008) revealed that fertilization with $\mathrm{N}$ may be used to reduce some of the negative effects of salinity. In addition, results of Rezvani Moghaddam et al. (2011) on wheat, Cerda and Martinez (1988) on tomato indicated that $\mathrm{N}$ had a positive effect 
on germination and emergence factors under saline stress and in disagreement with Fallahi and Khajeh-Hosseini (2011).

The root and shoot lengths are the most important parameters for salt stress because roots are in direct contact with soil and absorb water from soil and supply it to the rest of the plant.

In this study salinity affected the root and shoot length and dry weight, as root and shoot length, and TDW decreased when salinity increased (Tables 3,4 , and 5, Figures 2a, 3a, and 5a). Salinity reduced root and shoot lengths as the level of salt increased, however, the decrease of the root length was more prominent as compared shoot length. The reduction in the root and shoot lengths may be due to the toxic effect of $\mathrm{NaCl}$ as well as to an imbalance of the nutrient amount in the plant. Some studies indicate that the plant has a reduction in its growth due to the proportional increase of $\mathrm{Na}$. High salinity may inhibit root and shoot elongation due to the lower water uptake by the plant. Werner and Finkelstein (1995), and Kaya et al. (2003) observed that the root growth was more adversely affected as compared to shoot growth by salinity. The reduction in total dry weight was also finer in varieties having higher EP and shoot length (Tables 2 and 3). Our results are in agreement with the results from other researchers. For example, Hussein et al. (2007), Kochak-Zadeh et al. (2013), and Nimir et al. (2014) reported that a negative relationship was detected between vegetative growth parameters and increasing salinity also similar finding has been reported by Soltani and Galeshi (2002), Soltani et al. (2006), and Nimir et al. (2015). They found that seedling dry weight reduction in response to salinity was a result of reduction in seed reserve mobilization, not con version efficiency of mobilized reserve to seedling tissue. In this study interaction, results showed that $\mathrm{N}$ promoted the seedling growth such as root and shoot length, and TDW. At the different levels of salinity showed that $\mathrm{N}$ exerts a positive or ineffective effect on the growth parameters (Tables 3, 4, and 5). This is in agreement with previous reports in wheat (Fallahi \& Khajeh-Hosseini, 2011).

The varieties differed in their response to salinity, and their difference was attributed to the genetic variation (Hussien Ibrahim et al., 2016). For this reason root and shoot length provides an important clue for the plant response to salt stress (Jamil \& Rha, 2004). On the other hand, reduction in plant growth as a result of salt stress has also been reported in several other plant species (Ashrai \& McNelly, 1990; Mishra et al., 1991; Ashraf \& OLeary, 1997; Carpýcý et al., 2009). The increase in $\mathrm{NaCl}$ concentration decreased the shoot and root length and total dry weight of all the wheat varieties. Similar results were recorded by Hussein et al. (2007) and Carpýcý et al. (2009) in maize (Zea mays L).

As the salt concentrations increased, the STI and SVI of varieties decreased. As a result, the lowest values on the STI and SVI were determined at $200 \mathrm{mM} \mathrm{NaCl}$ (Tables 7 and 8). These results were in agreement with those of Javid et al. (2011), Carpýcý et al. (2009), Hussien Ibrahim et al. (2016), and Homayoun (2011) and in disagreement with Rahman et al. (2008). However, N improved STI and SVI of all varieties at various levels of salinity stress (Tables 7 and 8).

\section{Conclusions}

Our study investigated the affects in characteristics of seedling growth of four varieties of wheat exposed to salinity and nitrogen. Salinity inhibited emergence percentage, and subsequent growth of shoot and root. In this study nitrogen had a positive effect on emergence percentage, and seedling growth. As expected the emergence percentage decreased with increasing salinity and increased with increasing $\mathrm{N}$ level. Among varieties, Elnilein had a highest EP, shoot length, seedling vigor index, total dry weight, and salt tolerance, indicating a higher tolerance to salinity. Thus, this variety could be cultivated on slightly saline soils. However, this could not be a guarantee for it to be salt-tolerant in later growth stages. Therefore, to recommend the cultivation of this wheat variety in the field, there must be additional research conducted at the whole plant growth stage. From the present study, it can be concluded that nitrogen management is important when the plant growth in the saline soil. Further study to examine the effect of nitrogen in varieties of wheat will help us to see if there is any relationship between nitrogen and salinity tolerance of the seeds during early growth stages.

\section{Acknowledgements}

This study was financially supported in part by Natural Science Foundation of China (31171483), Priority Academic Program Development of Jiangsu Higher Education Institution, and Fund for Returning Overseas Scholars (2012).

\section{References}

Abdoli, M., Saeidi, M., Azhand, M., Jalali-Honarmand, S., Esfandiari, E., \& Shekari, F. (2013). The effects of different levels of salinity and Indole-3-Acetic acid (IAA) on early growth and germination of wheat seedling. Journal of Stress Physiology \& Biochemistry, 9(4), 329-338. 
Abdul-Baki, A. A., \& Anderson, J. D. (1973). Vigor determination in soybean seed by multiple criteria. Crop Science, 13(6), 630-633. http://dx.doi.org/10.2135/cropsci1973.0011183X001300060013x

Akbarimoghaddam, H., Galavi, M., Ghanbari, A., \& Panjehkeh, N. (2011). Salinity effects on seed germination and seedling growth of bread wheat cultivars. Trakia Journal of Sciences, 9(1), 43-50.

Al-Harbi, A., Wahb-Allah, M., \& Abu-Muriefah, S. (2008). Salinity and nitrogen level affects germination, emergence, and seedling growth of tomato. International Journal of Vegetable Science, 14(4), $380-392$. http://dx.doi.org/10.1080/19315260802371369

Almansouri, M., Kinet, J.-M., \& Lutts, S. (2001). Effect of salt and osmotic stresses on germination in durum wheat (Triticum durum Desf.). Plant and Soil, 231(2), 243-254. http://dx.doi.org/10.1023/A:101037840 9663

Almodares, A., Hadi, M., \& Dosti, B. (2007). Effects of salt stress on germination percentage and seedling growth in sweet sorghum cultivars. Journal of Biological Sciences, 7(8), 1492-1495. http://dx.doi.org/ $10.3923 /$ jbs.2007.1492.1495

Ashraf, M., \& OLeary, J. (1997). Ion distribution in leaves of salt-tolerant and salt-sensitive lines of spring wheat under salt stress. Acta Botanica Neerlandica, 46(2), 207-217.

Ashrai, M., \& McNelly, T. (1990). Improvement of salt tolerance in maize by selection and breeding. Plant Breeding, 104(2), 101-107. http://dx.doi.org/10.1111/j.1439-0523.1990.tb00410.x

Atak, M., Kaya, M. D., Kaya, G., Çikili, Y., \& Çiftçi, C. Y. (2006). Effects of $\mathrm{NaCl}$ on the germination, seedling growth and water uptake of triticale. Turkish Journal of Agriculture and Forestry, 30(1), 39-47.

Carpýcý, E., Celýk, N., \& Bayram, G. (2009). Effects of salt stress on germination of some maize (Zea mays L.) cultivars. African Journal of Biotechnology, 8(19) 4918-4922.

Cerda, A., \& Martinez, V. (1988). Nitrogen fertilization under saline conditions in tomato and cucumber plants. Journal of Horticultural Science, 63(3), 451 458. http://dx.doi.org/10.1080/14620316.1988.11515878

Fallahi, J., \& Khajeh-Hosseini, M. (2011). Effects of applying various levels of nitrogen on parent plants on the resistance to salinity stress in achieved seeds in Triticum aestivum L. cv. Gaskojen at germination period. Journal of Agricultural Technology, 7(6), 1743-1754.

Guarda, G., Padovan, S., \& Delogu, G. (2004). Grain yield, nitrogen-use efficiency and baking quality of old and modern Italian bread-wheat cultivars grown at different nitrogen levels. European Journal of Agronomy, 21(2), 181-192. http://dx.doi.org/10.1016/j.eja.2003.08.001

Hampson, C. R., \& Simpson, G. (1990). Effects of temperature, salt, and osmotic potential on early growth of wheat (Triticum aestivum). I. Germination. Canadian Journal of Botany, 68(3), 524-528. http://dx.doi.org/10.1139/b90-072

Homayoun, H. (2011). Effect of $\mathrm{NaCl}$ salinity on wheat (Triticum aestivum L.) cultivars at germination stage. Advances in Environmental Biology, 1716-1721.

Hussein, M., Balbaa, L., \& Gaballah, M. (2007). Salicylic acid and salinity effects on growth of maize plants. Research Journal of Agriculture and biological Sciences, 3(4), 321-328.

Hussien Ibrahim, M. E., Zhu, X., Zhou, G., \& Ahmed Nimir, N. E. (2016). Comparison of Germination and Seedling Characteristics of Wheat Varieties from China and Sudan under Salt Stress. Agronomy Journal, 108(1), 85. http://dx.doi.org/10.2134/agronj15.0176

Jamil, M., \& Rha, E.-S. (2004). The effect of salinity $(\mathrm{NaCl})$ on the germination and seedling of sugar beet (Beta vulgaris L.) and cabbage (Brassica oleracea L.). Plant Resources, 7(3), 226-232.

Javid, M. G., Sorooshzadeh, A., Moradi, F., Sanavy, S. A. M. M., \& Allahdadi, I. (2011). The role of phytohormones in alleviating salt stress in crop plants. Australian Journal of Crop Science, 5(6), 726.

Kanai, M., Higuchi, K., Hagihara, T., Konishi, T., Ishii, T., Fujita, N., ... Tadano, T. (2007). Common reed produces starch granules at the shoot base in response to salt stress. New Phytologist, 176(3), 572-580. http://dx.doi.org/10.1111/j.1469-8137.2007.02188.x

Kaya, M. D., Ipek, A., \& Öztürk, A. (2003). Effects of different soil salinity levels on germination and seedling growth of safflower (Carthamus tinctorius L.). Turkish Journal of Agriculture and Forestry, 27(4), 221-227. 
Kochak-Zadeh, A., Mousavi, S.-H., \& Nejad, M. (2013). The effect of salinity stress on germination and seedling growth of native and breeded varieties of wheat. J. Novel Appl. Sci., 12, 703-709.

Llanes, A., Reinoso, H., \& Luna, V. (2005). Germination and early growth of Prosopis strombulifera seedlings in different saline solutions. World Journal of Agricultural Sciences, 1(2), 120-128.

Malhi, S., Grant, C., Johnston, A., \& Gill, K. (2001). Nitrogen fertilization management for no-till cereal production in the Canadian Great Plains: A review. Soil and Tillage Research, 60(3), 101-122. http://dx.doi.org/10.1016/S0167-1987(01)00176-3

Mansour, M. (2000). Nitrogen containing compounds and adaptation of plants to salinity stress. Biologia Plantarum, 43(4), 491-500. http://dx.doi.org/10.1023/A:1002873531707

Meloni, D., Gulotta, M., \& Martinez, C. (2008). Salinity tolerance in Schinopsis quebracho colorado: Seed germination, growth, ion relations and metabolic responses. Journal of Arid Environments, 72(10), 1785-1792. http://dx.doi.org/10.1016/j.jaridenv.2008.05.003

Mishra, S. K., Subrahmanyam, D., \& Singhal, G. S. (1991). Interrelationship between salt and light stress on primary processes of photosynthesis. Journal of Plant Physiology, 138(1), 92-96. http://dx.doi.org/ 10.1016/S0176-1617(11)80736-4

Moud, A. M., \& Maghsoudi, K. (2008). Salt stress effects on respiration and growth of germinated seeds of different wheat (Triticum aestivum L.) cultivars. World J. Agric. Sci., 4(3), 351-358.

Munns, R. (2002). Comparative physiology of salt and water stress. Plant, Cell \& Environment, 25(2), $239-250$. http://dx.doi.org/10.1046/j.0016-8025.2001.00808.x

Munns, R., \& Tester, M. (2008). Mechanisms of salinity tolerance. Annu. Rev. Plant Biol., 59, 651-681. http://dx.doi.org/10.1146/annurev.arplant.59.032607.092911

Neumann, P. M. (1995). Inhibition of root growth by salinity stress: Toxicity or an adaptive biophysical response? Structure and function of roots (pp. 299-304). Springer.

Nimir, N. E. A., Lu, S., Zhou, G., Guo, W., Ma, B., \& Wang, Y. (2015). Comparative effects of gibberellic acid, kinetin and salicylic acid on emergence, seedling growth and the antioxidant defence system of sweet sorghum (Sorghum bicolor) under salinity and temperature stresses. Crop and Pasture Science, 66(2), 145http://dx.doi.org/10.1071/cp14141

Nimir, N. E. A., Lu, S., Zhou, G., Ma, B. L., Guo, W., \& Wang, Y. (2014). Exogenous Hormones Alleviated Salinity and Temperature Stresses on Germination and Early Seedling Growth of Sweet Sorghum. Agronomy Journal, 106(6), 2305-2315. http://dx.doi.org/10.2134/agronj13.0594

Passioura, J., \& Gardner, A. (1990). Control of leaf expansion in wheat seedlings growing in drying soil. Functional Plant Biology, 17(2), 149-157. http://dx.doi.org/10.1071/PP9900149

Qiu, N., Chen, M., Guo, J., Bao, H., Ma, X., \& Wang, B. (2007). Coordinate up-regulation of VH+-ATPase and vacuolar $\mathrm{Na}+\mathrm{H}+$ antiporter as a response to $\mathrm{NaCl}$ treatment in a $\mathrm{C} 3$ halophyte Suaeda salsa. Plant Science, 172(6), 1218-1225. http://dx.doi.org/10.1016/j.plantsci.2007.02.013

Rahman, M., Soomro, U., Haq, M. Z.-U., \& Gul, S. (2008). Effects of NaCl salinity on wheat (Triticum aestivum L.) cultivars. World Journal of Agricultural Sciences, 4(3), 398-403.

Rezvani Moghaddam, P., Fallahi, J., Khajeh Hosseini, M., Amiri, M. B., Aghhavani Shajari, M., \& Yazdani Biuki, R. (2011). The study of nutritional management of parent plant in combination with seed priming by biofertilizers in toward to increase of salinity tolerance in wheat cv. Sayonz at germination period. In 10th Conference of the International Society for seed Science.

Saboora, A., Kiarostami, K., Behroozbayati, F., \& Hajihashemi, S. (2006). Salinity (NaCl) tolerance of wheat genotypes at germination and early seedling growth. Pak. J. Biol. Sci., 9(11), 2009-2021. http://dx.doi.org/10.3923/pjbs.2006.2009.2021

Saunders, D. A., \& Hettel, G. P. (1994). Wheat in Heat-stressed Environments: Irrigated, Dry Areas, and Rice-wheat Farming Systems. Proceedings of the International Conferences: Wheat in Hot, Dry, Irrigated Environments, Wad Medani, Sudan, February 1-4, 1993, Wheat in Warm Area, Rice-wheat Farming Systems, Dinajpur, Bangladesh, Feburary 13-15 [sic], 1993. CIMMYT.

Shirazi, M., Asif, S., Khanzada, B., Khan, M., \& Mohammad, A. (2001). Growth and ion accumulation in some wheat genotypes under $\mathrm{NaCl}$ stress. Pak J Biol Sci, 4, 388-391. http://dx.doi.org/10.3923/pjbs.2001.388.391 
Soltani, A., \& Galeshi, S. (2002). Importance of rapid canopy closure for wheat production in a temperate sub-humid environment: Experimentation and simulation. Field Crops Research, 77(1), 17-30. http://dx.doi.org/10.1016/S0378-4290(02)00045-X

Soltani, A., Gholipoor, M., \& Zeinali, E. (2006). Seed reserve utilization and seedling growth of wheat as affected by drought and salinity. Environmental and Experimental Botany, 55(1), 195-200. http://dx.doi.org/10.1016/j.envexpbot.2004.10.012

Werner, J. E., \& Finkelstein, R. R. (1995). Arabidopsis mutants with reduced response to $\mathrm{NaCl}$ and osmotic stress. Physiologia Plantarum, 93(4), 659-666. http://dx.doi.org/10.1111/j.1399-3054.1995.tb05114.x

Xiong, F., Yu, X., Zhou, L., \& Wang, Z. (2013). Effect of Nitrogen Application at the Booting Stage on Wheat Progeny Seed Germination and Seedling Growth. Journal of Plant Studies, 2(2), 158. http://dx.doi.org/10.5539/jps.v2n2p158

Zhang, H., Irving, L. J., McGill, C., Matthew, C., Zhou, D., \& Kemp, P. (2010). The effects of salinity and osmotic stress on barley germination rate: sodium as an osmotic regulator. Annals of Botany, 106(6), 1027-1035. http://dx.doi.org/10.1093/aob/mcq204

Zhang, H., Zhang, G., Lu, X., Zhou, D., \& Han, X. (2014). Salt tolerance during seed germination and early seedling stages of 12 halophytes. Plant and Soil, 388(1-2), 229-241. http://dx.doi.org/10.1007/ s11104-014-2322-3

Zhou, G., Ma, B., Li, J., Feng, C., Lu, J., \& Qin, P. (2010). Determining salinity threshold level for castor bean emergence and stand establishment. Crop Science, 50(5), 2030-2036. http://dx.doi.org/10.2135/cropsci2009. 09.0535

Zhu, J.-K., Hasegawa, P. M., Bressan, R. A., \& Bohnert, H. J. (1997). Molecular aspects of osmotic stress in plants. Critical Reviews in Plant Sciences, 16(3), 253-277. http://dx.doi.org/10.1080/07352689709701950

\section{Copyrights}

Copyright for this article is retained by the author(s), with first publication rights granted to the journal.

This is an open-access article distributed under the terms and conditions of the Creative Commons Attribution license (http://creativecommons.org/licenses/by/4.0/). 